\title{
Sepsis with Streptoccocus pneumoniae in a child with nephrotic syndrome
}

\author{
Iulia Armean', Lorena Elena Melit ${ }^{1,2}$, Carmen Duicu1,2 \\ ${ }^{1}$ Pediatrics Clinic, Emergency Clinical County Hospital, Tg. Mures \\ ${ }^{2}$ University of Medicine and Pharmacy, Tg. Mures
}

\begin{abstract}
Introduction. Nephrotic syndrome is one of the most frequent glomerular pathologies encountered in pediatric ages, with an incidence of 4.7 cases in 100,000 children. The most frequent infectious complication of nephrotic syndrome is bacterial peritonitis caused most of the times by Streptococcus pneumoniae, a microorganism responsible by a high number of pneumonia, otitis media and sepsis episodes among children. Case report. We present the case of a 4.7-year-old male patient, diagnosed with nephrotic syndromes with 2 recurrences and transitory hypertension, admitted in our clinic for: diffuse abdominal pain, vomiting, diarrhea, fever and right otalgia. The laboratory tests on the day of admission pointed out elevated inflammatory biomarkers, leukocytosis, anemia and combined dyslipidemia. The urinary exam showed massive proteinuria. The blood culture was positive for Streptococcus Pneumoniae. The abdominal ultrasound revealed mild ascites, the thoracic radiography ruled out a pneumonia, and the ENT exam pointed out erythematous and bulged tympanic membrane. We initiated empirical therapy with Ceftriaxone, steroids and symptomatic drugs. After 3 weeks of hospitalization, the patient is discharged in good general status, with remission of nephrotic syndrome.

Conclusions. Congestive acute otitis media can lead in case of immunosuppressed patients to a severe sepsis with Streptococcus pneumoniae. Thus, anti-pneumococcal vaccination is mandatory, especially in case of high risk patients.
\end{abstract}

Keywords: nephrotic syndrome, sepsis, otitis media, Streptococcus pneumoniae

\section{INTRODUCTION}

Nephrotic syndrome (NS) is a frequently encountered pathology in nephrology clinics in both developed and developing countries, representing one of the most frequent glomerular disorders in pediatric ages (1-3). The incidence of NS worldwide is of 4.7 cases in 100,000 children, and the prevalence is estimated at $16 / 100.000$ children below the age of 16 years $(2,4)$. Classically, it is defined as massive proteinuria $\left(>40 \mathrm{mg} / \mathrm{m}^{2} / \mathrm{h}\right)$ responsible of hypoalbuminemia $(<2,5 \mathrm{~g} / \mathrm{dl})$ and generalized edema (1,5-9). Most of the cases of NS that sensitive to corticosteroids, but nonetheless approximately $20 \%$ of the cases are refractary to corticosteroids, frequently due to segmental focal glomerulosclerosis. These cases are characterized by the rapid progression towards chronic renal failure requiring dialysis and renal transplantation within the first years after diagnosis $(2,4)$. In idiopathic $\mathrm{NS}$, the most frequent histological finding is minimal changes glomerulonephritis ( $80 \%$ of cases) or segmental focal glomerulosclerosis. Other histological types of NS encountered in children include membranoproliferative glomerulonephritis and less frequently membranous nephropathy $(6,4,3)$.

Many times, severe complications are associated with NS mainly due to the massive losses of plasma proteins (1). The urinary loss of low molecular proteins such as: immunoglobulin $G$, factor I and factor B can lead to the alteration of opsonization, phagocytosis and the entire process of bacterial elimination resulting to immunosuppression of NS patients (10). 
The complications of NS are divided into two main categories: complications due to the disease itself and complications associated to therapy. The first category includes infections (peritonitis, sepsis, cellulitis, and chicken-pox), embolism (venous embolism and pulmonary embolism), hypovolemia (abdominal pain, tachycardia and hypotension), hyperlipemia, chronic renal failure, anemia etc. (1). It is estimated that approximately $1.5 \%$ of the cases of NS in children die as a consequence of infections associated to the underlying disease (11). Of this category, the most frequent complication is spontaneous bacterial peritonitis found in 1.5 to $16 \%$ of the cases (10). Most of the cases of peritonitis are caused by encapsulated gram positive bacteria, especially Streptococcus Pneumoniae that represent also a major cause of meningitis, pneumonia, acute otitis media and sepsis among children $(10,12,13)$.

Systemic inflammatory response includes at least two of the following criteria: tachypnea, bradycardia, hyperthermia or hypothermia and leukocytosis or leukopenia $(14,15)$.

Sepsis is defined as life-threatening organ dysfunction caused by a dysregulated host response to infection, being one of the most important causes of death worldwide, with an increasing incidence. According to the Society of Critical Care Medicine and the European Society of Intensive Care Medicine in 2016 was established that organ dysfunction can be represented by an increase in the Sequential Organ Failure Assessment (SOFA) which is associated with an in-hospital mortality greater than $10 \%$. Quick SOFA(qSOFA) is less robust than a SOFA score, it does not require laboratory tests and can be assessed quickly and repeatedly and should be used prompt to further investigate for organ dysfunction and to initiate or escalate therapy as appropriate (16-18).

\section{CASE PRESENTATION}

\section{Reasons of admission}

We present the case of a 4.7-year-old male child admitted to our clinic for the following complaints: diffuse abdominal pain, vomiting, diarrhea, fever, right otalgia. The personal pathological history revealed that the patient was diagnosed with NS with two episodes of relapse and transient hypertension. We mention that the patient had a poor socio-eco- nomic status, is unvaccinated, with inappropriate vitamin supplementation, and had extremely low compliance to treatment.

\section{Clinical exam}

At the time of admission, the clinical exam revealed the following pathological elements: altered general status, pale and shiny skin, generalized edema whit positive dimple sign at the level of pretibial area, right otalgia, tachycardia 146/min, hypertension $115 / 78 \mathrm{mmHG}$, tachypnea $30 / \mathrm{min}$, high fever $39,7^{\circ} \mathrm{C}$, distended abdomen, abdominal tenderness at palpation, vomiting, diarrhea, weight $17 \mathrm{~kg}$, height $107 \mathrm{~cm}$.

\section{Diagnostic assessment}

The laboratory tests on the day of admission pointed out: anemia (hemoglobin of $10.7 \mathrm{~g} / \mathrm{dl}$, hematocrit $31 \%$, mean erythrocyte volume $77.1 \mu \mathrm{L}$ ), leucocytosis (leukocytes $21800 \mu \mathrm{l}$ ), neutrophilia (neutrophils $70.6 \%$ ), thrombocytes $150,000 / \mathrm{mm}^{3}$; elevated inflammatory biomarkers (CPR $58.8 \mathrm{mg} /$ dl, ESR $87 \mathrm{~mm} / \mathrm{h}$ ); hyponatremia ( $\mathrm{Na} 132.2 \mathrm{mmol} / \mathrm{l}$, $\mathrm{K} 3.97 \mathrm{mmol} / \mathrm{l})$; hypercholesterolemia $(412 \mathrm{mg} / \mathrm{dl})$, while the values of triglycerides were within normal ranges. Also, the liver and renal functions proved to be normal. The urinary exam revealed: leukocyturia, hematuria $(50 / \mu 1)$, massive proteinuria $(500 \mathrm{mg} / \mathrm{dl})$ and the semi quantitative proteinuria was $>4500 \mathrm{mg} / \mathrm{l}$. qSOFA score was 3, respiratory rate $30 / \mathrm{min}$, altered general status with Glasgow coma scale 13, hypertension 115/78 mmHG (stage I according to gender and high). A thoracic radiography was performed which ruled out a possible pneumonia. The blood culture was positive for Streptococcus Pneumoniae, and the antibiogram showed susceptibility to: Penicillin, Amoxicillin, Cefotaxime, Ceftriaxone, Imipenem, Levofloxacin, Moxifloxacin, Ofloxacin, Erythromycin, Linezolid, Vancomycin, Tetracycline, Chloramphenicol, Rifampicin and Trimethoprim/Sulfamethoxazole.

The abdominal ultrasound revealed: hepatomegaly, nephromegaly, a fine blade of perirenal fluid and mild ascites. The surgical consult ruled out a spontaneous bacterial peritonitis. The ENT exam pointed out erythematous, bulged tympanic membrane, establishing the diagnosis of acute congestive otitis media. 


\section{Therapeutic assessment}

Thereby, following the clinical, paraclinical examination and qSOFA score a diagnosis of sepsis with Streptococcus pneumoniae as a result of acute congestive otitis media, relapsed nephrotic syndrome and anemia were established. Empirical therapy with Ceftriaxone, corticosteroids (methylprednisolone by vein) and symptomatic drugs was initiated, the evolution being favorable. After 3 weeks of hospitalization, the patient was discharged in good general status, in remission (without proteinuria) with the following recommendations: unsalted, hypoglycemic and hypolipemic diet, tapered oral corticosteroids, and adjuvant therapy with potassium supplements, proton pump inhibitors and $\mathrm{Ca}+$ Vitamin D3 supplements.

\section{DISCUSSIONS}

Among the pediatric population, the most common cause of NS is idiopathic, being diagnosed in $90 \%$ of children with aged between 1 to 10 years and in $50 \%$ of children diagnosed after above the age of 10 years (7). The incidence of this disease is reported between 2-7/100,000 children bellow 15 years of age, but the prevalence is higher due to the recurrent nature of this pathology (7). The incidence varies according to gender, age, ethnicity and environment, being more common among male patients, with a sex ratio of 1:3 for boys, and it may occur at any age but most frequently with onset between 2 and 7 years $(8,18)$. Similar to the data reported by the literature, the case presented above is a male child whose diagnosis of NS was established at approximately 3 years of age.

Infections are the main complications that threaten the lives of NS patients, due in particular to Streptococcus pneumoniae, the first cause of bacterial peritonitis and sepsis in these patients (9).

However, other microorganisms such as $\beta$ hemolytic streptococcus, Haemophilus and Gram negative bacteria are also commonly found (1). Thus, there were reported cases of infectious associations in patients with NS, such as the simultaneous presence of peritonitis, cellulitis and sepsis (10). Moreover, there were described also cases of sepsis with Vibrio cholerae, bacteria that causes mainly diarrhea, but due to severe immunosuppres- sion associated to NS it can lead to life-threatening disorders in these patients (19).

Otitis media (OM) is one of the most common pathologies encountered in pediatric age, and one of the most common causes of presentation to pediatrician and otorhinolaryngology specialist $(13,20)$. Among the complications of this pathology, sepsis is not routinely included, our patient being immunocompromised due to the underlying pathology, thus presenting sepsis due to otitis media.

Children diagnosed with SN should be vaccinated according to the national schedule and in addition they should be vaccinated against varicella zoster virus (3), but also Streptococcus pneumonia (9). Immunization is an effective method of preventing pneumococcal infections in children, however, for those diagnosed with $\mathrm{SN}$, vaccination is quite controversial, recent studies showing that vaccination is not as effective as in healthy children or it might induce relapses (9).

Most guidelines recommend anti-pneumococcal immunization using the 13-valent Pneumococcal Conjugated Vaccine, possibly followed by a dose of 23-valent Pneumococcal Polysaccharide Vaccine, the latter being administered only after 2 years of age (9).

The patient presented was not vaccinated according to the national scheme, thus being more susceptible to develop bacterial infections. Moreover, anti-pneumococcal vaccination would also be of real importance in this case.

\section{CONCLUSIONS}

Sepsis is a major cause of mortality and morbidity worldwide, with an increasing incidence.

Although this is not among the common complications of otitis media, we present the case of a child with a poor socio-economic status who associates NS with two relapses (not being compliant to treatment), complicated with congestive acute otitis media, leading to sepsis with Streptococcus pneumoniae. qSOFA score should be used prompt in order to asses organ dysfunction and to initiate therapy. We mention the importance of immunization in children, both with the vaccines included in the national schedule but in the case of those diagnosed with SN also with anti-pneumococcal vaccine. 


\section{REFERENCES}

1. Park S.J., Shin J.I. Complications of nephrotic syndrome. Korean J Pediatr. 2011; 54:322-328.

2. Gbadegesin R., Winn M., William E. Genetic testing in nephrotic syndrome: challenges and oportunities. Nat Rev Nephrol. 2013; 9:179-184.

3. Lennon R., Watson L., Webb N.J. Nephrotic syndrome in children. Paediatr child health. 2009; 20:36-42.

4. Sibley M., Roshan A., Alshami A., Catapang M., Jöbsis J. et al. Induction prednisone dosing for childhood nephrotic syndrome: How low should we go? Pediatr Nephrol. 2018 doi.org/10.1007/ s00467-018-3975-6.

5. Ulinski T., Aoun B. Pediatric idiopathic nephrotic syndrome: Treatment. Curr Med Chem. 2010; 17:847-853.

6. Obiagwu P.N., Aliyu A., Atanda A. Nephrotic syndrome among children in Kano:A clinicopathological study. Niger J Clin Pract. 2014; 17:370-374.

7. Mroczkowska K.E., Rzypczyk P., Tomaszewska M.P. Levamisole therapy in children with frequently relapsing and steroid-dependent nephrotic syndrome: a single-center experience. Cent Eur J Immunol. 2016; 41:243-247.

8. Boyer O., Baudouin V., Bérard E., Dossier C., Audard V. et al. Idiopathic nephrotic syndrome. Arch Pediatr. 2017; 24:1338-1343.

9. Pittet L.F., Posfay-Barbe K., Chehade H., Rudin C., Wilhelm-Bals A. et al. Optimizing seroprotection against pneumococcus in children with nephrotic syndrome using the 13-valent pneumococcal conjugate vaccine. Vaccine. 2016; 34:4948-4954.

10. Naseri M. Pneumococcal Sepsis, Peritonitis, and Cellulitis at the First Episode of Nephrotic Syndrome. IJKD. 2013;7:404-407.

11. Lagos L., Valero J. Coexistence of cellulitis and primary peritonitis in a pediatric patient with nephrotic syndrome: A case report. J Pediatr Surg Case Rep. 2017; 20:48-50.

12. Schnappauf C., Rodloff A., Siekmeyer W., Hirsch W., Sorge I. et al. Invasive pneumococcal diseases in children and adolescents-a single centre experience. BMC Research Notes. 2014; 7:145-152.
13. Lixandru R.I., Falup-Pecurariu C., Bleotu L., Mercas A., Leibovitz E. et al. Streptococcus pneumoniae Serotypes and Antibiotic Susceptibility Patterns in Middle Ear Fluid Isolates During Acute Otitis Media and Nasopharyngeal Isolates During Communityacquired Alveolar Pneumonia in Central Romania. Pediatr Infect Dis J. 2017; 36:151-154.

14. Meliț L.E., Mărginean C.O., Georgescu A., Duicu C. Complications of sepsis in infant. A case report. JCCM. 2016; 2:96-99.

15. Armean I., Duicu C., Aldea C., Melit L.E. Serratia marcescens Sepsis in a Child with Deep Venous Thrombosis - A Case Report. JCCM. 2018; 4:29-33.

16. Hawiger J., Veach R.A., Enki Ewicz J.Z. New paradigms in sepsis: From prevention to protection of failing microcirculation. J Thromb Haemost. 2015;13:1743-1756.

17. Hamers L., Kox M., Pickkers P. Sepsis-induced immunoparalysis: Mechanisms, markers, and treatment options. Minerva Anestesiologica. 2015; 81:426-439.

18. Singer M., Deutschman S.C., Seymour C.W., Shankar-Hari M., Djillali A. et al. The Third International Consensus Definitions for Sepsis and The Third International Consensus Definitions for Sepsis and. JAMA. 2016; 23:801-810.

19. Niaudet $P$., Boyer $O$. Idiopathic nephrotic syndrome in children:clinical aspects. Pediatr Nephrol. 2016; 7:839-882.

20. George N., Fredrick F., Mohapatra A., Veeraraghavan B., Kakde S.T. et al. Non-01, non-0139 Vibrio cholerae sepsis in a patient with nephrotic syndrome. Indian J Nephrol. 2013; 23:378-380.

21. Rettig E., Tunkel D.E. Contemporary Concepts in Management of Acute Otitis Media in children. Otolaryngol Clin North Am. 2014; 47:651-672. 